\title{
Dor facial atípica: caracterização de uma amostra
}

Manoel Jacobsen Teixeira*, Edson Bor Seng Shu**, José Cláudio Marinho da Nóbrega***, Cláudio Fernandes Corrêa****

Departamento de Neurologia da Faculdade de Medicina da Universidade de São Paulo

\section{RESUMO}

As características funcionais e morfológicas da face, aliadas à complexidade do fenômeno doloroso, tornam a face favorável à existência de inúmeras fontes de dor como procuram demonstrar as várias classificações existentes a respeito.

No período de janeiro de 1986 a março de 1993, 57 doentes com dor facial de etiologia incerta foram submetidos a avaliações neurológica, bucomaxilofacial, psiquiátrica, psicológica e de medicina física. Foram também investigados, por procedimentos de imagem do crânio, coluna cervical e exames de laboratório, incluindo o do líquido cefalorraquidiano. A idade média dos doentes foi 51 anos. Trinta e nove doentes (68\%) eram do sexo feminino. O tempo de história da dor variou de 1 mês a 23 anos, com média de 61,8 meses. A dor em queimação foi a mais freqüente (43\%) seguida de latejante (33\%). A hemiface direita foi acometida em 27 doentes $(47,3 \%)$ e 8 doentes $(14,2 \%)$ tiveram comprometimento bilateral. Quanto à distribuição topográfica de acometimento houve predomínio em V2, com 17 doentes (29,8\%); a hemiface inteira foi comprometida em 10 doentes (17,5\%). Irradiação para locais não correspondentes ao território de inervação do nervo trigêmeo observou-se em 15 doentes (26,3\%), sendo em 5 (33,3\%) para a região cervical, em 5 (33\%) para o ouvido, em 2 (13,3\%) irradiou para a região occipital e em outros 2 para a nuca. A dor teve freqüência diária em 43 doentes $(75,4 \%)$. A duração da dor tomava a maior parte do dia em todos os doentes.

Fatores moduladores de melhora ou piora da dor facial atípica foram encontrados em 27 doentes (47,3\%); desses, 4 (14,8\%) referiram melhora da dor com pressão ou calor local e 23 (85\%) referiram fatores de piora da dor, sendo o nervosismo e o frio os principais fatores relatados, respectivamente, por $10(17,6 \%)$ e $8(14 \%)$ dos doentes. Dois (3,5\%) pacientes pioraram com o calor. Fenômenos neurovegetativos não foram raros, acometendo 20 doentes (35\%). Sintomas neuropsíquicos, depressão elou ansiedade foram encontrados em $28(49,1 \%)$ doentes. A síndrome dolorosa miofascial esteve associada em 18 (31,5\%) doentes. Vinte e um doentes (36,8\%) tiveram antecedentes de trauma ou cirurgia da face. Alterações no exame físico foram observadas em 13 (22,8\%) doentes, sendo 11 (19,3\%) doentes com hipoestesia facial.

A investigação diagnóstica dos 57 pacientes com padrão de dor facial atípica confirmou 45 casos de dor facial atípica. Em 12, outros diagnósticos foram encontrados.

O diagnóstico de dor facial atípica é de exclusão e a avaliação multiprofissional é essencial.

\section{PALAVRAS-CHAVE}

Dor facial. Dor facial atípica.

\section{ABSTRACT}

Atypical facial pain: characterization of a sample

The complexity of pain and the functinal and morphologic peculiarities of the face justify a variable sources of facial pain.

Fifty-seven patients with facial pain of uncertain etiology were evaluated, from January 1986 to November 1993. All the patients were submited to neurologic, maxilo-facial, psyquiatric, psychological and physiatric evaluation. Complementary investigation included radiological, neuroimaging and laboratorial procedures. The median age was 51 years. Thirty nine (68\%) were female. The onset of the symptoms had occurred 1 month to-23 year before this study (medium 61,8 months). The most frequent charactheristics of pain were the burning (43\%) and the throbing (33\%). The pain was refered on the rigth in $27(47,3 \%)$ and was bilateral in $8(14,2 \%)$. The predominant distribution was the V2, found in 17 patients $(29,8 \%)$ and the whole hemiface was compromised in $10(17,5 \%)$ patients. In $15(26,3 \%)$ the irradiation of pain was extratrigeminal: cervical in $5(33,3 \%)$, to the ear in $5(33,3 \%)$, occipital in $2(13,3 \%)$ and nuchal in $2(13,3 \%)$. The pain was diary in $43(75,4 \%)$, in the most of patients its duration take all the day long. Local heat and compression alleviated the pain in $4(14,8 \%)$. Twenty three (85\%) refered worsening agents, the cold and psycological stresses being the most frequent and cited by $18(17,6 \%)$ and $8(14 \%)$

\footnotetext{
* Professor Doutor do Departamento de Neurologia da Faculdade de Medicina da Universidade de São Paulo.

** Residente de Neurocirurgia do Hospital das Clínicas da Faculdade de Medicina da Universidade de São Paulo.

*** Neurocirurgião Pesquisador do Departamento de Neurologia da Faculdade de Medicina da Universidade de São Paulo.

**** Neurocirurgião do Serviço de Neurocirurgia Funcional do Instituto de Psiquiatria da Faculdade de Medicina da Universidade de São Paulo.
} 
patients, respectively. Neurovegetative symptoms were present in 20 (35\%). Depression and/or anxiety was present in 28 (49\%). Myofascial pain was present in $18(31,5 \%)$. Twenty-one patients $(36,8 \%)$ have had surgical or traumatic antecedents in the face. Abnormal clinical findings were found in 13 (22,8\%), facial hypoestesia being the most frequent and detected in $11(19,3 \%)$.

At the end of investigation, 45 patients could be classified as suffering of atypical facial pain. In 12, other pathologies were diagnosed. Therefore it is concluded that a multiprofissional evaluation is very important for this entity.

\section{KEYWORDS}

Atypical facial pain. Facial pain.

\section{Introdução}

Qualquer dor de cabeça é considerada algia craniofacial, incluindo as chamadas cefaléias primárias, ou seja, aquelas não associadas a lesões anatômicas, e as relacionadas a lesões estruturais e constantes incluídas na Classificação Internacional de Cefaléias.

As características funcionais e morfológicas da face, aliadas à complexidade do fenômeno doloroso, tornam favorável a existência de inúmeras fontes de dor, como procuraram demonstrar as várias classificações existentes a respeito ${ }^{28}$.

A caracterização e o diagnóstico diferencial das dores craniofaciais são complexos e incluem doenças cuja origem primária não está sediada na face. Portanto, o diagnóstico exige história e exame clínico minuciosos. O modo de apresentação, o caráter, a localização, o padrão, os fatores de melhora e piora e os sinais e os sintomas associados podem ser de grande valia no diagnóstico. Resultados de exames complementares (radiografias simples, tomografia computadorizada, ressonância magnética), de avaliações otorrinolaringológicas, odontológicas, bucomaxilofaciais e, eventualmente, da execução de bloqueios anestésicos diagnósticos devem ser cuidadosamente analisados.

A dor facial pode ser decorrente de três mecanis$\operatorname{mos}^{9}$ : neuropáticos, originados de qualquer lesão de elementos do sistema nervoso periférico ou central; nociceptivos, decorrentes de estímulos nociceptivos resultantes de lesões teciduais, ou serem resultantes de fenômenos desconhecidos.

Loeser $^{18}$, em 1985, descreveu a neuralgia facial típica como uma síndrome dolorosa restrita à distribuição de um nervo craniano específico ou de seus ramos. Pode surgir em qualquer nervo que contenha fibras aferentes somáticas como os nervos trigêmeo, intermédio, glossofaríngeo, vago e, raramente, nas raízes dorsais cervicais de $\mathrm{C}_{2}$ e $\mathrm{C}_{3}$.

Distinguem-se três padrões de dor facial trigeminal neuropática ${ }^{5}$ : a neuralgia clássica (ou típica) do nervo trigêmeo, a neuropatia trigeminal e a neuralgia atípica do trigêmeo. A mais comum das neuralgias típicas é a neuralgia clássica do trigêmeo. Teixeira ${ }^{27}$ caracterizou a neuralgia clássica do nervo trigêmeo por cinco aspectos básicos: é paroxística, apresenta fatores desencadeantes definidos, limita-se ao território de distribuição do nervo trigêmeo, acomete um lado da face, não se associa a anormalidades do exame neurológico e a anormalidades dos exames complementares.

O termo neuralgia facial atípica foi criado por Frazier e Russel ${ }^{8}$, em 1924, para denominar a dor facial difusa, sem causa aparente e sem as características da neuralgia essencial do nervo trigêmeo e do glossofaríngeo.

Stookey e Ransohoff ${ }^{26}$, em 1959, classificaram a neuralgia facial atípica em duas categorias. Numa, a dor é restrita à porção medial da face, região maxilar, frontal ou globo ocular, podendo sugerir neuralgia da divisão maxilar ou oftálmica do quinto nervo. A região mandibular raramente é comprometida. Na segunda categoria, a dor localiza-se nas áreas de distribuição de outros nervos cranianos ou cervicais. Irradia-se para as regiões frontal, occipital, retromastóidea, cervical e, às vezes, para o ombro.

White e Sweet ${ }^{29}$ classificaram, como neuralgia facial atípica, a dor facial constante e profunda, não limitada ao território do nervo trigêmeo, com duração de horas, dias ou meses, ocorrendo sem fatores precipitantes, em doentes com tendência à dependência de drogas e com personalidade neurótica.

Em 1981, Cusik ${ }^{4}$ conceituou a neuralgia facial atípica como dor facial vaga, com fisiopatologia desconhecida e com distribuição que não respeita o território de inervação dos nervos sensitivos da face. Intensifica-se com o frio, a fadiga, a ansiedade e a depressão ${ }^{26}$. Não há fatores desencadeantes, como ocorre na neuralgia típica ${ }^{15}$. É contínua, dura horas ou dias. Raramente se instala ou desaparece subitamente ${ }^{26}$. Os doentes com dor facial atípica costumam apresentar traços neuróticos ${ }^{25}$.

As neuralgias atípicas formam um grupo diverso das dores faciais. Loeser ${ }^{18}$ descreveu-as como dores que, algumas vezes, não respeitam a distribuição de um nervo, podem ser bilaterais, intra-orais ou faciais e podem estender-se até o pescoço; são contínuas, usualmente com discreto déficit sensitivo, ausência de pontos-gatilho e, com freqüência, ocorrem em pacientes 
com psicopatologias. Loeser ${ }^{19}$ classificou as dores faciais atípicas em quatro tipos. No primeiro tipo, a dor é constante, usualmente em queimação, restrita à hemiface algumas vezes dor em choque ou em punhaladas superpõe a dor de fundo. Antecedente de traumas na face são comuns e, eventualmente, infecções ou neoplasias estão associadas. Bloqueios do nervo ou procedimentos ablativos, freqüentemente, não resultam em melhora. No segundo tipo, a dor inicia-se insidiosamente e aumenta de intensidade e distribuição em meses; alterações de sensibilidade não são observadas no início, entretanto, com a progressão, hipoestesia torna-se evidente. A dor é profunda, dolorida, algumas vezes em queimação, e as zonas-gatilho estão presentes raramente. Yonas e Jannetta acreditam que esse tipo de dor provavelmente esconde neoplasias ou infecções da base do crânio ( apud Loeser $^{19}$ ). Um outro tipo associa-se com uma ampla variedade de fenômenos autonômicos. $\mathrm{O}$ último tipo ocorre sem nenhum antecedente de trauma. O paciente queixa-se de queimação constante geralmente circunscrita. Não há perda sensorial. Mulheres jovens são a maioria dos acometidos e a dor facial atípica costuma ser bilateral. Alterações comportamentais, freqüentemente, antecedem o início do quadro álgico.

Em 1988, a Sociedade Internacional de Cefaléias ${ }^{13}$ apresentou a classificação e os critérios diagnósticos das cefaléias, neuralgias cranianas e dor facial. Nessa calssificação, 13 grupos são catalogados. O grupo 11 é composto por cefaléia ou dor facial associada com patologias do crânio, pescoço, orelha, olhos, nariz, seios, dentes, boca, ou outras estruturas faciais ou cranianas e, no grupo 12, encontram-se neuralgias cranianas, dor de troncos nervosos e dor por desaferentação. A partir disso, a dor facial persistente sem as características das neuralgias cranianas descritas e que não se associa a sinais físicos ou a uma causa orgânica demonstrável passou a denominar-se "dor facial que não preenche os critérios dos grupos 11 e 12" em substituição ao termo "dor facial atípica".

Dores faciais que não se enquadram nos critérios pertencentes ao item 12.8 da Classificação Internacional de Cefaléias (CIC) são denominadas genericamente de dores faciais atípicas ${ }^{1,11,12,24}$. São dores persistentes na face, que não preenchem os critérios das neuralgias e das síndromes dolorosas anteriormente descritas e não são associadas a sinais neurológicos objetivos e nem a lesões orgânicas demonstráveis nos exames de imagem. A dor geralmente é confinada a uma área limitada de uma hemiface (pode ser bilateral em 20\% a 35\% dos casos), comumente na região maxilar, podendo espraiar-se para a região mandibular e cervical. É profunda e de localização imprecisa. Pode manifestar-se após cirurgias ou traumatismos da face, dentes e gengivas, mas persiste sem causa evidente. Predomina no sexo feminino e na quarta década de vida. Embora a dor seja descrita como muito intensa e contínua, os doentes não expressam esse sofrimento. A resposta terapêutica costuma ser insatisfatória. A dor é notavelmente resistente aos analgésicos, inclusive morfina. Usualmente, há piora após certos procedimentos (bloqueio anestésico, cirurgia dos seios da face, rizotomia). Os doentes habitualmente requerem insistentemente tais procedimentos pela convicção de que "alguma coisa causa a dor" e, caracteristicamente, refutam as bases psicológicas. Todos autores concluem que é uma condição eminentemente psicogênica; praticamente todos os doentes apresentam anormalidades da personalidade como depressão, ansiedade, transtorno obsessivocompulsivo, histeria, síndrome pós-traumática ou uma combinação dessas. Em 50\% dos casos, o início é atribuído a um evento físico como cirurgias, extrações dentárias, traumatismos da face, dentes ou gengiva e problemas emocionais. Poucos casos melhoram com a psicoterapia. A despeito das conotações psicofisiológicas, a dor facial atípica apresenta várias qualidades comuns às dores por desaferentação, como o aspecto difuso e a melhora com antidepressivos tricíclicos. Especula-se haver deficiência da atividade serotoninérgica central e depleção de opióides endógenos nesses casos. Muitos doentes com dor por disfunção da articulação temporomandibular apresentam características psicológicas semelhantes aos com dor facial atípica; diversos autores consideram alguns desses casos como formas localizadas da mesma condição, assim como a chamada "odontalgia atípica", particularmente quando ocorrem disestesias orais traduzidas por queimor na boca e na língua. O diagnóstico de dor facial atípica deve ser feito após a exclusão de afecções orgânicas pelo exame físico, neurológico de imagem e de laboratório. A dor oculta originada nas cavidades dentárias infectadas pode mimetizá-la e requerer bloqueios anestésicos alveolares seletivos para sua identificação. Também pode simular a anestesia dolorosa trigeminal e dor facial talâmica. A "dor facial póstraumática" e a "dor dentária fantasma", após extração dentária, não são reconhecidas pela CIC e confundem-se com a dor facial atípica e a "odontalgia atípica".

Esse trabalho objetivou caracterizar uma amostra de 57 doentes com diagnóstico inicial de dor facial atípica quanto aos aspectos demográficos, clínicos e de exames complementares. Para o propósito, uma revisão da literatura foi realizada e confrontada com nossa casuística.

\section{Casuística e método}

Esse trabalho é baseado na análise de 57 pacientes adultos, de ambos os sexos, com padrão de dor facial 
atípica, acompanhados na Liga de Cefaléia e Algias Craniofaciais do Centro Acadêmico Oswaldo Cruz e do Departamento de Neurologia do Hospital das Clínicas da Faculdade de Medicina da Universidade de São Paulo, no período de janeiro de 1986 a dezembro de 1993.

Todos os doentes foram submetidos a avaliações odontológica, neurológica, otorrinolaringológica, fisiátrica, psicológica, psiquiátrica e à investigação por procedimentos de imagem do crânio, coluna cervical e exames de laboratório, incluindo o do líquido cefalorraquidiano.

A semiologia odontológica foi aferida por meio de um protocolo especialmente organizado na Divisão de Odontologia do Hospital das Clínicas da Faculdade de Medicina da Universidade de São Paulo (HCFMUSP); a avaliação neurológica e a otorrinolaringológica, segundo o protocolo de dor do Centro de Dor do Hospital das Clínicas da FMUSP; as qualidades da dor, segundo o questionário de dor de McGill; as anormalidades radiológicas do esqueleto facial (radiografias panorâmicas do crânio, face e coluna cervical e tomografia computadorizada do crânio) segundo a normatização da Divisão de Odontologia do HCFMUSP; a avaliação psicocomportamental, segundo entrevistas semiestruturadas e testes psicológicos (Beck e IDATE).

\section{Resultados}

A idade dos doentes variou entre 22 e 86 anos, com média de 51 anos. Trinta e nove (68\%) doentes eram do sexo feminino. O tempo de história da dor variou de 1 mês a 23 anos, com média de 61,8 meses.

Quanto ao caráter da dor, o descritor "em queimação" foi o mais freqüente (29,1\%), seguido de "latejante" $(24,1 \%)$ (Tabela 1$)$. A hemiface direita foi acometida em 27 doentes (47,3\%), e 8 deles (14,2\%) tiveram comprometimento bilateral (Figura 1).

Quanto à distribuição topográfica de acometimento, houve predomínio de V2 com 17 doentes (29,8\%); havia comprometimento dos três ramos do nervo trigêmeo em dez doentes (17,5\%) (Figura 2). Irradiação para locais não correspondentes ao território de inervação do nervo trigêmeo observou-se em 15 doentes $(26,3 \%)$ : em 5 doentes (33,3\%) para a região cervical, em $5(33,3 \%)$ para a orelha, em $2(13,3 \%)$ para a região occipital, 2 para a nuca e 1 doente $(6,8 \%)$ apresentou irradiação para a região temporal (Tabela 2 ).

A dor teve freqüência diária em 43 doentes (75,4\%). A duração da dor atingia a maior parte do dia em todos os doentes.

Fatores moduladores de melhora ou piora da dor facial atípica foram encontrados em 27 doentes (47,3\%);

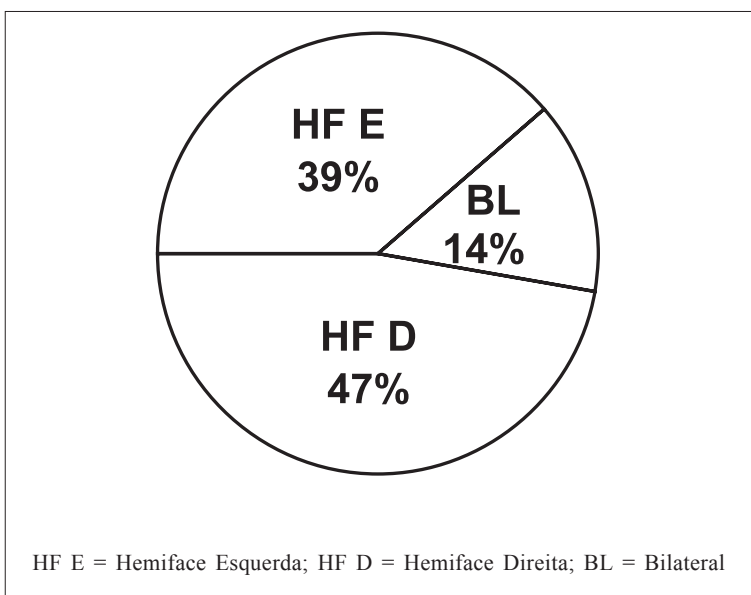

Figura 1 - Distribuição dos doentes por território facial acometido.

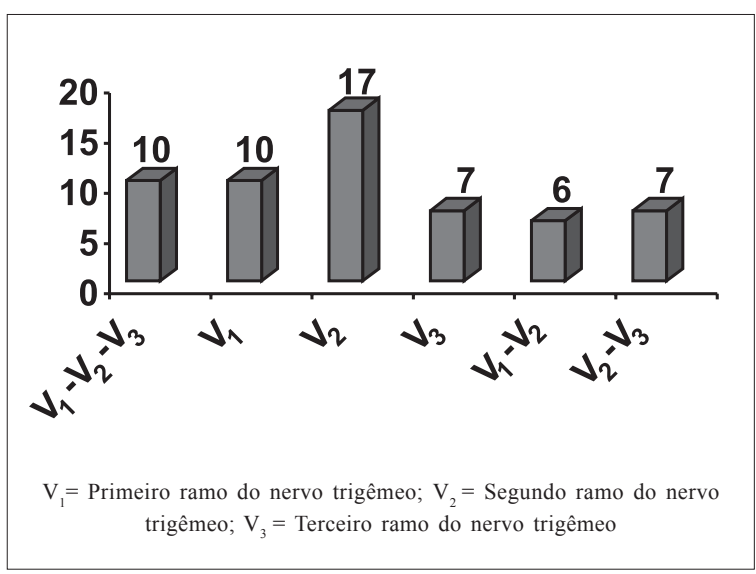

Figura 2 - Distribuição de doentes segundo a localização da dor.

desses, $23(85 \%)$ referiram fatores de piora da dor, sendo o nervosismo e o frio os principais fatores e indicados, respectivamente, por $10(17,6 \%)$ e $8(14 \%)$ dos doentes; 4 doentes $(14,8 \%)$ referiram melhora da dor com pressão ou calor local. Fenômenos neurovegetativos não foram raros, acometendo 20 doentes (35\%) (Tabela 3 ).

Sintomas neuropsíquicos, depressão e/ou ansiedade foram encontrados em $28(49,1 \%)$ doentes. A síndrome dolorosa miofascial esteve associada em 18 $(31,5 \%)$ doentes. Vinte e um doentes $(36,8 \%)$ tiveram antecedentes de trauma ou cirurgia da face. Alterações no exame físico foram observadas em $13(22,8 \%)$ doentes; $11(19,3 \%)$ doentes apresentavam hipoestesia facial.

A investigação diagnóstica dos 57 pacientes com padrão de dor facial atípica confirmou 45 casos de dor facial atípica e, em 12, outros diagnósticos foram encontrados (Tabela 4). 


\begin{tabular}{lcc}
\hline \multicolumn{3}{c}{ Tabela } \\
& Incidencia dos padrões de dor \\
\hline Padrão da dor & $\mathbf{N}^{\mathbf{0}}$ de casos & $\%$ \\
\hline Queimação & 23 & 29,1 \\
Latejante & 19 & 24,1 \\
Pontada & 8 & 10,1 \\
Choque & 8 & 10,1 \\
Agulhada & 8 & 10,1 \\
Peso & 5 & 6,3 \\
Aperto & 4 & 5,1 \\
Formigamento & 4 & 5,1 \\
\hline Total & 79 & 100 \\
\hline
\end{tabular}

\begin{tabular}{lc}
\hline & $\begin{array}{c}\text { Tabela } \\
\text { Irradiação }\end{array}$ \\
\hline Irradiação da dor & \\
\hline Região cervical & $\mathbf{N}^{\mathbf{0}}$ de doentes \\
Orelha & 5 \\
Nuca & 5 \\
Occipital & 2 \\
Temporal & 2 \\
\hline Total & 1 \\
\hline
\end{tabular}

\begin{tabular}{|c|c|}
\hline \multicolumn{2}{|c|}{$\begin{array}{c}\text { Tabela } 3 \\
\text { Fenômenos neurovegetativos associados }\end{array}$} \\
\hline Sinais e sintomas & $\mathrm{N}^{0}$ de casos \\
\hline Lacrimejamento & 8 \\
\hline Hiperemia conjuntival & 5 \\
\hline Obstrução nasal & 3 \\
\hline Edema local & 3 \\
\hline Salivação abundante & 1 \\
\hline Total & 20 \\
\hline
\end{tabular}

\begin{tabular}{|c|c|c|}
\hline \multicolumn{3}{|c|}{$\begin{array}{c}\text { Tabela } 4 \\
\text { Diagnósticos encontrados em } 57 \text { pacientes com } \\
\text { padrão de dor facial atípica }\end{array}$} \\
\hline Diagnósticos & $\mathbf{N}^{0}$ de casos & $\%$ \\
\hline Dor facial atípica & 45 & 78,9 \\
\hline Meningeoma & 1 & 1,8 \\
\hline Invaginação vertebrobasilar & 1 & 1,8 \\
\hline Neurolues & 1 & 1,8 \\
\hline Hipoestesia facial & 9 & 15,7 \\
\hline Total & 57 & 100 \\
\hline
\end{tabular}

\section{Discussão}

Na literatura, a variação da idade desses doentes com dor facial atípica é ampla (20 a 82 anos) com média de $51 \operatorname{anos}^{19}$. Na nossa casuística encontramos uma variação de 22 a 86 anos com média igual ao trabalho citado. Todas as publicações indicam o predomínio das mulheres $(82 \%$ a $100 \%)$ nos $\operatorname{casos}^{10,19} ; 68 \%$ da nossa casuística eram do sexo feminino.

Law e Nelson ${ }^{17}$ descreveram-na como dolorimento, compressão, latejamento, repuxão, queimor e aperto difuso ou circunscrito. Na nossa investigação, padrão de dor em queimação foi o mais freqüente, sendo encontrados 23 casos $(29,1 \%)$, que estão de acordo com a literatura ${ }^{5,10,18,19,22,30}$. Os demais estavam distribuídos entre sete padrões diferentes (Tabela 1).

Todos os doentes apresentaram comprometimento da área de inervação do nervo trigêmeo, conforme mostrado (Gráfico 2). A divisão V2 foi a mais comprometida, observada em 17 doentes $(29,8 \%)$; o comprometimento simultâneo dos três ramos juntos e o comprometimento isolado de $\mathrm{V} 1$ tiveram igual distribuição, com 10 doentes $(17,5 \%)$ cada um; dor abrangendo simultaneamente os territórios V2 e V3 foi observada em 7 doentes (12,3\%). Essa apresentação topográfica contrasta com a distribuição típica observada nos doentes com neuralgia essencial do trigêmeo, em que a segunda e a terceira divisões juntas são as mais comumente acometidas; raramente a primeira divisão do trigêmeo é acometida, o que ocorre em apenas 7\% dos casos de neuralgia essencial ${ }^{18,27}$.

A dor facial atípica pode irradiar-se para as regiões retroauricular, cervical, frontal, occipital e do ombro ${ }^{26,32}$. Rabelo $^{22}$ ressalta a importância de avaliar a possibilidade de patologias torácicas (neoplasias pulmonares), determinando dores com caráter de dor facial atípica, por mecanismo de dor referida. Em consonância com a literatura, 15 doentes $(26,3 \%)$ do nosso estudo apresentaram irradiação para locais não correspondentes ao território de inervação do trigêmeo. Os locais mais comuns foram a região cervical e a orelha, ambas com cinco doentes (Tabela 2).

A dor facial atípica é contínua dura horas ou dias. Raramente se instala ou desaparece subitamente ${ }^{32}$. A dor teve freqüência diária em 43 doentes $(75,4 \%)$. A duração da dor compreendia a maior parte do dia, em todos os doentes, intensifica-se com o frio, a fadiga, a ansiedade e a depressão $0^{26}$. Fatores moduladores de melhora ou piora da dor facial atípica foram encontrados em 27 doentes (47,3\%); desses, $4(14,8 \%)$ referiram melhora da dor com pressão ou calor local e 23 (85\%) doentes referiram piora da dor, sendo o nervosismo e o frio os principais fatores, com respectivamente 10 $(17,6 \%)$ e $8(14 \%)$ dos doentes. Dois $(3,5 \%)$ pacientes referiram piora com o calor. 
Presença de fenômenos neurovegetativos acompanhando o quadro álgico é controverso na literatura. Yair ${ }^{30}$ descreve como fenômeno não usualmente observado. Stookey e Ransohoff ${ }^{26}$ relatam que pode ser acompanhada de lacrimejamento, rinorréia e hiperemia da face. Na nossa casuística foi observado, em 20 doentes (35\%): lacrimejamento foi o fenômeno mais comum e observado em 8 (40\%); somente $1(5 \%)$ doente apresentou salivação abundante (Tabela 3).

Várias publicações têm sugerido que a dor facial atípica seja uma desordem psiquiátrica ${ }^{6,7,16,23,30}$. Depressão é considerado o mais provável diagnóstico ${ }^{40}$.

Burchiel e cols ${ }^{3}$. descreveram que achados psicológicos tais como desilusões, alucinações, doentes com múltiplas queixas, conversão clássica ou pseudosintomas neurológicos, relatos de sintomas exagerados, depressão, procura excessiva de medicamentos ou tratamentos, preocupação excessiva e medo de sintomas estão presentes em pacientes com dor facial atípica. Em 28 de nossos doentes $(49,1 \%)$ foram encontrados sintomas de depressão e/ou ansiedade. Isso corrobora com os achados da literatura e orienta a necessidade de acompanhamento psiquiátrico desses doentes.

Síndrome dolorosa miofascial (SDM) é uma disfunção muscular regional causada pela presença de pontos-gatilho no músculo esquelético ${ }^{14}$. A característica clínica básica da SDM consiste em dor localizada, associada a bandas musculares tensas, palpáveis, nas quais encontramos um ponto miálgico intensamente dolorido, o ponto-gatilho. Yeng ${ }^{31}$ relata que de 164 indivíduos com dor craniofacial e cervical examinados em clínicas odontológicas, 55\% apresentavam dor em pontos-gatilho miofasciais. A SDM não diagnosticada, e conseqüentemente não tratada, causa sintomas dolorosos crônicos, podendo levar ao diagnóstico errôneo e ao insucesso no tratamento. Em 18 doentes $(31,5 \%)$ do nosso estudo foi encontrada SDM, patologia importante a ser considerada devido à alta freqüência com que ela se associa às dores neuropáticas e também pela necessidade de seu tratamento para lograrmos sucesso terapêutico.

Dor facial atípica pode iniciar-se após cirurgias ou trauma da face, dentes ou gengivas, porém, persistem sem qualquer causa demonstrável ${ }^{19,22}$. Na nossa casuística, em 21 doentes $(36,8 \%)$ esses achados estavam presentes.

Após a investigação diagnóstica do grupo estudado, foram encontrados 45 doentes $(78,9 \%)$ com dor facial caracterizada com a que não preenche os critérios dos grupos 11 e 12. Outros diagnósticos concluídos foram hipoestesia facial em 9 doentes $(15,7 \%)$, meningioma em $1(1,8 \%)$, neurolues em outro e invaginação vertebrobasilar noutro.
Em nosso meio, Okada e cols. ${ }^{21}$ estudaram 33 doentes com dor facial atípica e constataram anormalidades neurológicas em $63,5 \%$ dos casos (neuropatia traumática em 42,4\%; neuropatia idiopática em $21 \%$ ). Esses autores encontraram neoplasias em dois doentes $(3,3 \%)$, sendo um tumor do ângulo pontocerebelar e outro tumor do assoalho da boca. Outros relatos de tumor e dor facial atípica têm sido descritos ${ }^{2,20}$.

\section{Conclusão}

As características funcionais e morfológicas da face, aliadas à complexidade do fenômeno doloroso, tornam favorável a existência de inúmeras fontes de dor facial. A dor facial de padrão atípico envolve uma variedade de doenças locais e sistêmicas, sendo fundamentais anamnese e o exame clínico minuciosos, associados à investigação complementar. O diagnóstico da dor facial que não preenche os critérios dos grupos 11 e 12 é de exclusão, sendo a avaliação multiprofissional essencial.

\section{Referências}

1. BOGDUK N: Pain of cranial nerve and cervical nerve origin others than primary neuralgias. In Olesen $\mathrm{J}$, Tfelt-Hansen P, Welch KMA (ed): The headaches. New York, Raven Press, 1993, pp 765-72.

2. BULLITT E, TEW JM, BOYD J: Intracranial tumors in pacients with facial pain. J Neurosurg 64:865-71, 1986.

3. BURCHIEL KJ, BURGESS JA: Differential diagnosis and manangement of orofacial pain. In Tolisson CD, Satterthwaite JR, Tolisson JW (ed): Handbook of pain management. 2 ed. Philadelphia, Willians \& Wilkins, 1994, cap 22, pp 280-93.

4. CUSIK JF: Atypical trigeminal neuralgia. JAMA 245:2328-9,1981.

5. DE MARCO JK: Trigeminal neuropathy. Neurosurg Clin North Am 8:103-30, 1989.

6. ENGEL GL: Primary atypical facial neuralgia. An hysterical conversion symptom. Psychosomatic Med 13:375, 1951.

7. FEINMANN C, HARRIS M, CAWLEY R: Psychogenic facial pain: presentation and treatment. Brit Med $J$ 288:436, 1984

8. FRAZIER CH, RUSSEL EC: Neuralgia of the face. Arch Neurol Psychiatry (Chicago) 11:557-63,1924.

9. FROMM GH: Trigeminal neuralgia and related disorders. Neurol Clin 7:305-19,1989.

10. GALVÃO ACR: Diagnóstico diferencial das algias craniofaciais: condutas em neurologia. São Paulo, Academia Brasileira de Neurologia, 1997, pp 95105.

11. GOUDA JJ, BROWN JA: Atypical facial pain and others pain syndromes: differential diagnosis and treatment. Neurosurg Clin North Am 4:87-100, 1997. 
12. GRAFF-RADFORD SB, SOYKA D: Headache related to oromandibular structures. In Olesen J, Tfelt-Hansen P, Welch KMA (ed): The headaches. New York, Raven Press, 1993, pp 759-63.

13. HEADACHE CLASSIFICATION COMMITTE OF THE INTERNATIONAL HEADACHE SOCIETY: Classification and diagnostic criteria for headache disorders, cranial neuralgias and facial pain. Cephalalgia 8 (suppl 7):196, 1988.

14. IASP: International Association for the Study of Pain Subcommitte on Taxonomy: Classification of chronic pain, description of chronic pain syndromes and definitions of pain terms. Pain 3 (suppl):S1-S225, 1986.

15. KIRK EJ: Impulses in dorsal spinal nerve rootlets in cats and rabbits arising from dorsal ganglia isolated from the periphery. J Comp Neurol 155:165-76, 1974.

16. LASCELLES RG: Atypical facial pain and psychiatry. Brit J Psychiatry 112:654, 1966.

17. LAW WR, NELSON ER: Internal carotid aneurysm as a cause of Reader's paratrigeminal syndrome. Neurology (Chicago) 18:43-6, 1968.

18. LOESER JD: Cranial neuralgias. In Bonica JJ, Loeser JD, Chapman CR, Fordyce WE (ed): The management of pain. 2 ed. Philadelphia, Lea \& Febiger, 1994, vol 1, cap 38, pp 684-5.

19. LOESER JD: Tic Douloureux and atypical face pain. In Wall PD, Melzack R (ed): Textbook of pain. 3 ed. London, Churchill Livingstone, 1994, cap 39, pp 699710 .

20. NGUYEN M, MACIEWICZ R, BOUCKOMS A, POLETTI C, OJEMANN R: Facial pain symptoms in patients with cerebellopontine angle meningioma and rewiew of the literature. Clin J Pain 2:3, 1986.

21. OKADA M, TEIXEIRA MJ, LIN TY, AMARAL TGF, SIQUEIRA JT, FORMIGONI FG, PIMENTA CA, OLIVEIRA MF: Atyppical facial pain. A multidisciplinary assessement. Arq Neuropsiquiat 18:233, 1998.

22. RABELLO GD: Cefaléias primárias mais comuns. In Siqueira JTT, Ching LH (ed): Dor orofacial/ATM. Bases para o diagnóstico clínico. Curitiba, Ed Maio Ltda, 1999, pp 95-105.
23. REMICK RA, BLASBERG B: Psychiatric aspects of atypical facial pain. J Can Dental Assoc 12:913, 1985.

24. SOLOMON S, LIPTON RB: Facial pain. Neurol Clin 8:913- 8, 1990

25. SPECULAND B: Intractable facial pain and illnesss behavior. Pain 11:213-9, 1981.

26. STOOKEY B, RANSOHOFF J: Trigeminal neuralgia. Its history and treatment. Springfield, Charles C Thomas, 1959.

27. TEIXEIRA MJ: Critérios de dor facial atípica. In Anais do $3^{\circ}$ Simpósio Internacional de Dor, São Paulo, 1997, pp 19.

28. TEIXEIRA MJ, OKADA M: Dores atípicas da face. In Siqueira JTT, Ching LH (ed): Dor orofacial/ATM. Bases para o diagnóstico clínico. Curitiba, Ed Maio Ltda, 1999, pp 143-5.

29. WHITE JC, SWEET WH: Pain and the neurosurgeon. Springfield, Charles C Thomas Publisher, 1959.

30. YAIR S: Orofacial pain. In Wall PD, Melzack R (ed): Textbook of Pain. 3 ed. London, Churchill Livingstone, 1994, pp 575-82.

31. YENG LT, KAZIYAMA HHS, TEIXEIRA MJ: Síndrome dolorosa miofascial e fibromialgia. In Siqueira JTT, Ching LH (ed): Dor orofacial/ATM. Bases para o diagnóstico clínico. Curitiba, Ed Maio Ltda, 1999, pp 107-42.

32. YONAS H, JANNETTA PJ: Neurinoma of trigeminal root and atypical trigeminal neuralgia. Neurosurgery 6:273-7, 1987.

Original recebido em janeiro de 2000

Aceito para publicação em março de 2000

\section{Endereço para correspondência:}

Manoel Jacobsen Teixeira

Rua General Mena Barreto, 756

CEP 01433-010 - São Paulo, SP 